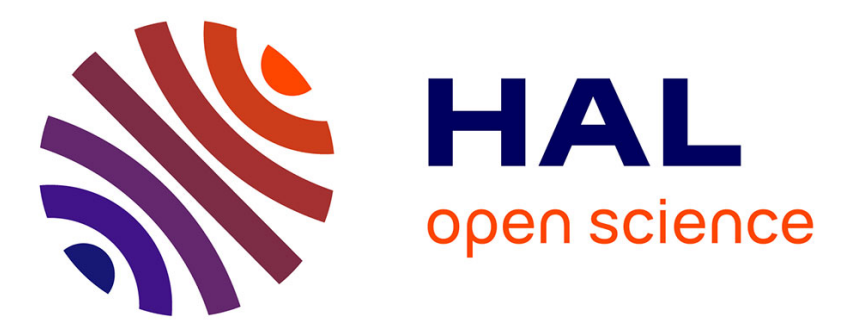

\title{
Histoires d'aiguilles chez les Kel Ahaggar. A propos d'un épisode méconnu du rituel du mariage
}

Paul Pandolfi

\section{To cite this version:}

Paul Pandolfi. Histoires d'aiguilles chez les Kel Ahaggar. A propos d'un épisode méconnu du rituel du mariage. Journal des Africanistes, 1994, 64 (1), pp.81 - 90. 10.3406/jafr.1994.2396 . hal-03174295

\section{HAL Id: hal-03174295 \\ https://hal.science/hal-03174295}

Submitted on 19 Mar 2021

HAL is a multi-disciplinary open access archive for the deposit and dissemination of scientific research documents, whether they are published or not. The documents may come from teaching and research institutions in France or abroad, or from public or private research centers.
L'archive ouverte pluridisciplinaire HAL, est destinée au dépôt et à la diffusion de documents scientifiques de niveau recherche, publiés ou non, émanant des établissements d'enseignement et de recherche français ou étrangers, des laboratoires publics ou privés. 


\section{Histoires d'aiguilles chez les Kel Ahaggar. A propos d'un} épisode méconnu du rituel du mariage

In: Journal des africanistes. 1994, tome 64 fascicule 1. pp. 81-90.

Citer ce document / Cite this document :

Pandolfi Paul. Histoires d'aiguilles chez les Kel Ahaggar. A propos d'un épisode méconnu du rituel du mariage. In: Journal des africanistes. 1994, tome 64 fascicule 1. pp. 81-90.

doi : 10.3406/jafr.1994.2396

http://www.persee.fr/web/revues/home/prescript/article/jafr_0399-0346_1994_num_64_1_2396 


\title{
Notes et documents
}

\author{
PAUL PANDOLFI
}

\section{Histoires d'aiguilles chez les Kel-Ahaggar A propos d'un épisode méconnu du rituel du mariage}

Dans la littérature concernant les Kel-Ahaggar, on peut trouver de nombreuses descriptions (plus ou moins précises, plus ou moins complètes) du rituel du mariage. Ainsi a-t-on relevé plusieurs fois ce moment particulier que l'on pourrait dénommer l'épisode des iratimen. Il a été signalé dès 1908 par M. Benhazera et, par la suite, nombre d'auteurs en ont proposé une explication ${ }^{1}$. De quoi s'agit-il exactement?

En Ahaggar, la cérémonie du mariage dure sept jours. Le premier soir, à la nuit tombée, la mariée et le groupe de femmes qui l'accompagne se dirige vers la tente nuptiale. Ce cortège féminin adopte alors une démarche extrêmement lente et entonne des chants spécifiques dénommés taré en tamâhaq. La mariée, quant à elle, le visage entièrement recouvert par un voile, est située au centre même de la procession, entourée, protégée par les femmes présentes. Au plus près d'elle, se trouvent des parentes et amies qui prennent garde à ce que personne ne puisse la toucher. Ce passage de la tente familiale à la tente nuptiale est présenté par les Daj-zali comme un moment fort critique durant lequel la mariée est particulièrement exposée aux éventuelles attaques des "génies " (Kel-Essuf) comme au " mauvais œil »(tehot). Aussi, est-ce pour empêcher tout contact qu'un groupe de femmes se presse autour de la mariée. De même, nombre d'entre elles agitent à bout de bras des brûle-parfums dans lesquels se consument des encens dont la fumée possède la propriété d'éloigner les Kel-Essuf.

L'abri nuptial n'est, ce premier soir, qu'une petite tente qui a été fort sommairement dressée sur un lit de sable de 50 centimètres de haut environ. Ce petit monticule, dénommé adebel, ne sera par la suite touché par personne. Plus longtemps, il restera en place, plus durable sera l'union célébrée ce soir-là. C'est sous la petite tente dressée sur cet adebel que se retrouvent, ce premier soir, les nouveaux époux. Le mari, accompagné par un cortège d'hommes chantant el-burda,

1. Outre M. Benhazera (1908: 17), on pourra se reporter aux quelques descriptions sommaires de cette coutume donnees par H. Lhote (1951:115 et 1984:186), J. Regnier (1961) et M. Chabrolles (1990: 115), ainsi qu'aux travaux plus importants de J. Nicolaisen (1962: 84) et surtout M. (jast (1973). 
y est arrivé le premier ${ }^{2}$. Son cortège a tourné trois fois, par la droite, autour de la tente avant que le marié, en prenant soin de poser le pied droit en premier, ne s'installe sous ce petit velum. Le cortège de la mariée fera, lui aussi, trois fois le tour de la tente nuptiale mais par la gauche cette fois-ci ${ }^{3}$. Mais, avant d'en arriver là, ce cortège féminin a dû, par un don d'iratimen, lever un obstacle.

En effet, quand la mariée et son escorte féminine se dirigent vers la tente nuptiale, elles sont interceptées en cours de route par un groupe de jeunes gens qui, avec force cris et gestes, leur barrent le passage. Ils réclament les itatimen qui sont des sandales de cuir fabriquées au Niger (notamment à In-Gall) et fort réputées en Ahaggar. Après un simulacre de dispute plus ou moins long, une ou deux paires d'itatimen sont offertes à l'un des opposants en l'occurrence le fils de la sœur du père de la mariée dénommé ababah ou tamâhaq ${ }^{4}$. Alors, et alors seulement, le groupe des jeunes gens se retire et le cortège de la mariée peut poursuivre son chemin vers la tente nuptiale.

Toute une part de jeu entre ici en compte. Cet aspect ludique tient aussi une grande part dans l'attachement des Kel-Ahaggar envers cette coutume. Ainsi, il ne faut point s'imaginer que le don des iratimen provoque immédiatement la levée de l'obstacle. Une fois les sandales offertes, tous les jeunes gens participant au barrage (mais aussi des spectateurs de la scène) se précipitent pour voir les izatimen, les examiner, les juger. En général s'élève alors une vive discussion pour savoir si ces sandales sont estimées ou non d'une qualité suffisante. Tout le monde prend parti, donne son avis y compris les femmes escortant la mariée. Il est d'aillcurs fréquent que pour accentuer cet aspect ludique, on offre intentionnellement - dans un premier temps - des iratimen de faible valeur voire même usagées ! Ces sandales seront alors rejetées avec force sarcasmes et ce n'est qu'après le renouvellement de la demande que l'on tendra à l'ababah des sandales répondant aux critères de qualité (épaisseur, qualité des coutures, finition...) connus de tous.

J. Nicolaisen, puis de manière plus précise encore M. Gast, ont montré comment cette coutume des iratimen peut être interprétée comme une véritable indemnité due par la mariée (et sa famille) à son ababah et plus précisément encore au fils de la sœur du père de la mariéc. Car c'est bien ce dernier qui, parmi les jeunes gens qui s'opposaient au passage du cortège, reçoit les itatimen".

Or, c'est lui aussi qui, si l'on s'en tenait au mariage préférentiel tel qu'il est énoncé dans le discours des Kel-Ahaggar, devrait se marier avec sa cousine croisée

2. El-burda est un poème composé au xil sièce par El-Bûsîrî en l'honneur du Prophète (Doutté 1984 : 217). Dans certaines régions musulmanes, es vers jouissent de proprićtés miraculeuses el sont récités lors de graves maladies. En Ahaggar, ce poème est systématiquement psalmodié par le cortege des hommes le premier soir du mariage. Selon N. Mecheri, cette coutume serait une pratique récente introduite il y a seulement une trentaine d'années par des tolba nouvellement installés en Ahaggar. Auparavant, seuls quelques amis accompagnaient le marié vers la lente (Mecheri $1986: 56$ ).

3. Ces connotations (droite-masculin / gauche-féminin) sont fréquentes che\% les Kel-Ahaggar : voir notamment la disposition spatiale du " mobilier " sous les tentes (Pandolfi 1989). Quant à la triple circumambulation, elle se retrouve che\% d'autres groupes touaregs (Casajus 1987:243), voire dans l'ensemble du monde berbère (I.aoust 1993).

4. En fait, le terme ibúbah (masculin : ababah, féminin : lababaht) désigne tous les cousins croisés d'Ego que ce soit du côté paternel ou du côté maternel. Pour des raisons de commodité de lecture, quand, dans la suite de notre texte, nous emploierons le terme ababah, c'est au scul cousin croisé patrilatéral de la mariée que nous nous réfèrerons.

5. On notera que sur ce point les observations des différents auteurs déjà cités sont loin d'être concordantes. Ainsi, selon M. Benhazera, c'est le fils de la tante maternelle de la mariéc qui stoppe le cortége et reclame les iratimen (1908: 17). Pour H. I.hote. c'est "le cousin germain par lignée utérine qui intervient (1984: 186). J. Regnier parle lui de l'oncle maternel de la mariéc (1961: 168) lout comme 
matrilatérale (tababaht). Lui offrir les iratimen afin de lever ce simulacre d'opposition au mariage prévu, c'est tout à la fois reconnaître cette priorité de l'ababah mais aussi l'annuler : le cousin croisé ayant reçu son dédommagement, sa cousine pourra dès lors se marier comme elle l'entend.

\section{Mariage préférenticl et don des sandales}

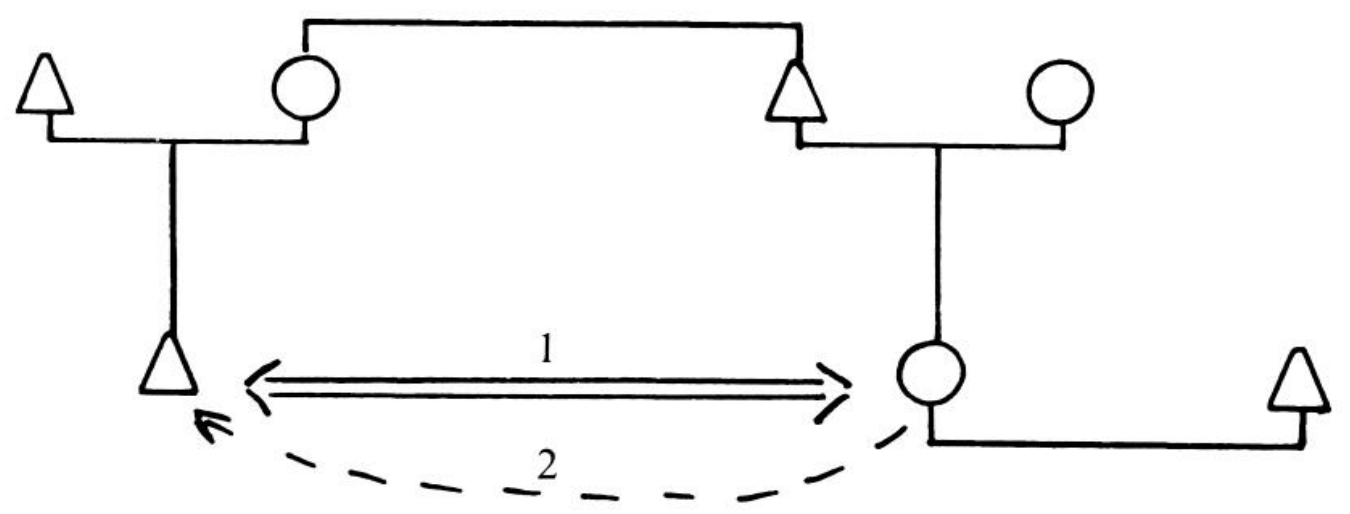

(1) mariage préférentiel

(2) don des sandales

Mais, si cet épisode des izatimen a déjà été décrit et commenté, il n'en est point de même d'un autre aspect du rituel qui - à notre connaissance - n'a, lui, jamais été relevé. Il s'agit de l'épisode des stenfassen qui se déroule le deuxième jour du mariage.

Le deuxième soir, en effet, à la nuit tombante, la jeune mariée accompagnée de nombreuses femmes se dirige à nouveau vers la tente où se trouve son époux. Cette tente n'est plus l'abri sommaire et provisoire qui avait été monté le premier soir au-dessus de l'adebel. Il s'agit maintenant d'une belle tente de cuir (ehen) qui, au matin de ce deuxième jour, a été dressée à distance respectable de l'abedel par les femmes du campement. A l'intérieur, le sol a été recouvert de beaux tapis et on y trouve exposés, non sans une certaine ostentation, tous les objets et ustensiles propres à la vie nomade (sacs de voyage, selle, isebran, coffre à thé...).

5. (suite) M. (habrolles qui précise qu'en l'absence de celui-ci, e'est la tante maternelle ou à defaut un parent maternel de la mariée qui peut s'opposer au passage du cortège et recevoir les sandales (1990: 115). Pour notre part, et au vu de ce que nous avons pu observer et entendre dans l'Ahaggar, nous rejoignons les dires de J. Nicolaisen (1962) et M. (iast (1973) pour qui c'est au cousin croisé patrilatéral de la mariée que reviennent en priorité les iratimen. Par l'emploi de cette expression, nous voulons spécifier que c'est là, nous semble-t-il, que se trouve la significátion première de ce don des sandales. Nous n'ignorons pas cependant que dans certaines circonstances c'est à une autre personne que le fills de la soeur du père de la mariée que sont dévolus les izatimen : don à un forgeron dans certains mariages Kel-zela (Gast 1973) oú, comme nous avons pu l'observer à Terhanânet, don à un membre des lseqqamaren lors de mariage Dag-tali, etc. 
Mais à nouveau, en cours de route, le cortège de la mariée est intercepté par un groupe de jeunes gens qui s'opposent à son avance. Cette fois-ci, ils réclament des stenfassen (awid stenfassen) terme qui en tamâhaq désigne les aiguilles (voir Foucauld 1951, IV : 1 904). Ce n'est que lorsque cette demande sera satisfaite que le cortège pourra reprendre son chemin. Le groupe de jeunes gens qui intervient ainsi est exclusivement composé d'esclaves (iklan) ou du moins, de nos jours, de personnes reconnues - et se reconnaissant - comme descendants d'esclaves. Selon nos informateurs (Dag-rali et Aguh-n-tahlé), il s'agissait autrefois essentiellement d'iklan appartenant au campement de la mariée et même s'ils pouvaient recevoir le renfort d'autres iklan présents à la cérémonie, c'est eux qui recevaient les stenfassen distribuées par les femmes du cortège.

De nos jours, ce sont des fils d'esclaves qui participent à cet épisode, du moins ceux qui acceptent d'y prendre part et donc d'endosser - ne serait-ce qu'un instant - cette étiquette dévalorisante. Il arrive d'ailleurs que cet épisode du rituel n'ait plus lieu aujourd'hui faute de participants! Tel ne semble point être le cas

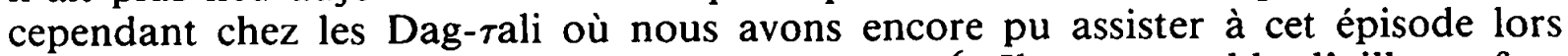
d'un mariage célébré en juin 1988 à Terhanänet ${ }^{6}$. Il nous semble d'ailleurs fort significatif qu'une telle coutume persiste encore de nos jours dans l'A haggar et que des jeunes gens qui bien souvent se sont intégrés dans la nouvelle structure sociale (travail salarié à Tamanrasset notamment) acceptent le temps d'une cérémonie de réendosser leur rôle d'iklan au vu et au su de tous. Cela démontre - si nécessaire - que les changements les plus évidents, les plus spectaculaires d'une société ne peuvent gommer d'un seul coup tout ce qui perdure du passé dans le présent.

Mais au-delà de ce constat, est-il possible de fournir une explication de cet épisode méconnu du rituel du mariage?

Dans la mesure où nous retrouvons là le schéma type de l'épisode des iratimen (simulacre d'opposition au mariage / cadeau-dédommagement / levée de l'opposition), il semble que l'on puisse utiliser pour les stenfassen la même logique du dédommagement que celle utilisée pour les iratimen. Les stenfassen apparaissant dès lors comme un don offert aux iklan par celle qui se marie et qui s'apprête à quitter le campement pour aller s'installer dans celui de son mari.

Pour une femme Kel-Ahaggar en effet, se marier signifie aussi (et peut-être même surtout) s'engager à changer de résidence. Certes ce changement n'est point immédiat puisque pendant une période de durée variable (mais rarement inférieure à un ou deux ans) la nouvelle mariée continuera à résider dans le campement de ses parents ${ }^{7}$. Mais, quoiqu'il en soit, le mariage s'inscrit déjà comme l'annonce, la préfiguration de cette rupture résidentielle. Les chants (âléwen) des femmes qui escortent la mariée vers la tente nuptiale l'énoncent clairement :

6. On pourrait se demander, vu le silence de la littérature ethnographique sur ce sujet, si cette coutume des stenfassen n'est pas récente dans l'A haggar. Bien qu'il nous soit impossible d'avancer une quelconque datation, nous sommes plutôt enclins à penser l'inverse. Nous avons observé ce rituel chez les Dagtali qui, pour diverses raisons que nous ne pouvons examiner dans le cadre de cet article, sont certainement le groupe (tawsit) le plus " conservateur » de l'Ahaggar. Quand nous avons évoqué cet épisode avec d'autres Kel-Ahaggar (notamment Kel-Ahnet), ils nous avouèrent en avoir entendu parler mais, fort étonnés que cela puisse encore exister, nous indiquèrent que chez eux cette coutume ancienne (ieru, ieru) avait disparu depuis longtemps.

7. Nous ne pouvons dans le cadre de cet article revenir sur toutes les règles et variantes du phénomène résidentiel chez les Kel-Ahaggar (Pandolfi 1990 : 150,173). Au-delà de cette complexité, deux phases essentielles se dégagent après le mariage : une première période (de durée variable) de résidence duolocale suivie d'une phase de résidence patrilocale. Le passage de la première à la seconde étant marqué par la fête de l'azalay (Chatelard 1990). 
Au nom de Dieu jusqu'à sept fois

Pour notre fille qui s'en va

Que la précède le nom de Dieu

Et de Mohammed jusqu'à la fin (Foucauld 1930, II, $n^{\circ} 516$ )

Le mariage étant présenté comme le dénouement du lien qui rattache la mariée à sa famille et à son campement :

J'ai dénoué le lien de la pouliche

De la pouliche née chez moi (ibid.)

Ainsi, les iklan voient partir une personne qui, vu les rapports domestiques qui régissaient souvent les relations entre hommes libres et esclaves dans les campements, pouvait représenter pour eux un appui, une source possible d'aide et d'éventuels « cadeaux ". Ils perdent alors une femme avec qui, suite à une vie résidentielle commune, s'étaient bien souvent établis des rapports de type familial. Certes, vu leur statut, cette perte ne peut être compensée - comme dans le cas des iratimen - par un cadeau luxueux. Aussi, ce sont des objets de moindre valeur qui sont ici offerts au iklan. En ce sens, la coutume des stenfassen répèterait celle des iratimen sur un mode mineur.

Si cette connotation quelque peu dévalorisante existe notamment dans le discours tenu sur ce sujet par les Kel-Ahaggar eux-mêmes, on ne peut oublier cependant que les aiguilles n'étaient point, en raison certainement de leur apparition tardive dans cette région, sans valeur. Outre de nombreuses mentions éparses dans la correspondance du père de Foucauld, on notera qu'il est souvent mentionné, dans les récits des Européens qui fréquentèrent les Kel-Ahaggar en ce début du siècle, que les aiguilles au même titre que le thé ou le tabac étaient toujours un cadeau fort apprécié.

Pour les petits dons, tels que le lait offert souvent aux étrangers, il n'est rien que les Iforas reçoivent plus volontiers en remerciement qu'un peu de tabac du Touat, ou encore quelques aiguilles à coudre (Cortier 1908: 337).

Je viens de recevoir la visite d'Aflane et Dacïne. Sans gêne, ils m'ont demandé le thé (il était 1 heure !)... Un cadeau de quinine et d'aiguilles, et les voilà partis contents (Vermale 1926 : 117).

Prestige qui subsiste encore de nos jours. En 1991, j'ai pu voir le beau-père d'un ami touareg revenant du marché du Tamanrasset. Il y avait acquis quelques fruits et légumes, des bonbons pour les jeunes enfants mais aussi un petit paquet d'aiguilles qu'il partagea fort équitablement entre sa femme et ses deux filles.

Dans le même ordre d'idées, Antoine Chatelard, qui partage depuis de nombreuses années la vie des Kel-Ahaggar, a bien voulu me transmettre ce témoignage :

J'ai rencontré vers 1965 au pied de l'Assekrem un homme qui revenait du Niger après une assez longue absence. Il m'a montré une boîte d'allumettes avec deux aiguilles piquées à l'intérieur. C'est un cadeau (tasiwit) d'un Tel pour une Telle, m'a-t-il dit, et admiratif, il ajouta : c'est un beau cadeau (tasiwit ti tularet) [Litt. 14 juin 1991].

Autant d'exemples qui nous incitent à ne point envisager les stenfassen sous le seul angle utilitaire mais à surtout voir en elles un don symbolique. 
Déjà, le père de Foucauld signalait dans ses Textes touaregs en prose que les femmes Kel-Ahaggar offraient des aiguilles comme porte-bonheur aux hommes partant en rezzou (1984: 287). On ne peut omettre également que comme nombre d'objets métalliques les aiguilles - à un degré moindre que les épées et poignards cependant - sont assez fréquemment utilisées pour éloigner les génies dans le monde berbère (Westermarck 1921: 132 et 143).

Mais il nous paraît surtout significatif que cet objet se retrouve dans les rituels qui marquent le début et la fin de la période (dénomméc asri en tamâhaq) durant laquelle une femme peut être courtisée. Dans l'Ahaggar, quand une jeune fille devient pubère, deux changements, l'un concernant sa coiffure, l'autre son apparence vestimentaire manifestent publiquement son nouvel état ${ }^{8}$.

Dès l'âge de 6-7 ans jusqu'à leurs premières règles, les jeunes filles adoptent un style de coiffure dénommé asakat en tamâhaq. Devenues pubères, elles abandonnent ce type de coiffure réservé aux jeunes adolescentes pour adopter celui des femmes adultes nommé émêsi ${ }^{9}$. Ce changement de coiffure est à tel point lié à la puberté que par extension le terme tamessekat désigne une « petite fille non pubère entre 7 et 14 ans " et que l'expression "... eg asakat (faire l'asakat, porter l'asakat) signifie être entre 7 et 14 ans et n'être pas encore pubères " (Foucauld 1951, IV : 1819 ).

Si la tête hirsute de l'enfant est signe de sa marginalité par rapport au monde des adultes, la coiffure apparaît souvent comme une des marques de la socialisation et il est fréquent qu'un changement de statut s'indique par l'adoption d'une nouvelle coiffure (Leach 1980 et Calame-Griaule 1981). C'est là un trait fréquent dans le monde berbère, mais, ce changement de la coiffurc féminine intervient généralement lors du mariage (Laoust 1993). Tel n'est point le cas ici : une des spécificités du monde touareg étant de ne point lier perte de la virginité et union matrimoniale.

Plus important encore que ce changement de coiffure est l'adoption par les jeunes filles pubères du voile dénommé èkerhei ${ }^{10}$. Ce dernier, porté par les femmes nomades de l'A haggar, consiste en une étoffe de couleur indigo d'un mètre de long sur 40 centimètres de large environ. Il se place sur la tête de manière à laisser le front et quelques centimètres de cheveux à découvert. Il retombe à droite et à gauche du visage laissant la face découverte mais cachant les oreilles et les faces latérales du cou. C'est le port de l'ékerhei qui manifeste sans ambages au vu de tous, qu'une jeune fille est désormais devenue une femme.

Pour célébrer cet événement, une fête avec chants (tindé), carrousels de chameaux (ilugan), etc., est organisée ${ }^{11}$. La jeune fille revêt un bel ékerhei dont les

8. En lamâhac, pour désigner les menstrues, on utilise l'expression îba $n$ amâd qui renvoie directement à la notion d'une impureté incompatible avec la pratique religicuse puisque signifiant "absence de la prière ". I.es lemmes, en effer, ne peuvent prier durant la période de leurs régles. De plus, elles représentent alors un danger pour toute forme ou manifestation d'al-haraka. Aussi outre la prière, elles s'absticndront alors de goûter au lait d'animaux ayant des petits, de boire l'eau contenue dans des outres récemment confectionnées, etc. (Nicolaisen 1961).

9. Pour de plus amples détails sur ces différents types de coiffure, on pourra se reporter à l'article émêsi dans le Dictionnaire touareg-français (Foucauld 1951, III : 1241 ). Dans les poésies touarègues, la coiffure est, avec les dents et les yeux, un des attraits principaux des femmes aimées (cf. notamment (ialand-Pernet $1978: 7$ ).

10. Il y a donc bien un terme spécifique, dans l'Ahaggar, pour désigner le voile porté par les femmes à la différence de ce qui se passe che\% les Kel-Ferwan de l'Aïr. Selon D. Casajus, c'est pour le seul voile masculin qu'est utilisé, tout comme dans l'Ahaggar, le terme tagelmust (Casajus 1987: 318). 11. L a prise du voile par les garçons est, elle aussi (quoique de manière moins directe), liée à la puberté mais elle ne s'accompagne point d'une fête semblable et s'opère - dans l'Ahaggar du moins - de manière beaucoup plus discrète. Cela n'est point le cas des Kel-Ferwan, puisque D. Casajus a relevé 
pans latéraux sont réunis sous son menton à l'aide d'une aiguille. Un des hommes présents à la cérémonie est alors désigné par les femmes présentes. L'homme ainsi élu doit être ce que l'on nomme en Ahaggar un ales wan tidit, c'est-à-dire un homme qui par sa conduite, actes et paroles, manifeste au mieux l'ethos touareg : maîtrise de soi, sens de l'honneur, élégance, bravoure...

Il peut arriver - comme cela nous a été signalé par certains de nos informateurs - que le choix des femmes se porte sur un jeune homme qui a déjà manifesté son intention de courtiser la jeune fille, voire sur un de ccux qui au vu des "préceptes » du mariage préférentiel dans le monde touareg est en bonne position pour obtenir sa main. Quoiqu'il en soit, c'est à l'élu de l'assemblée féminine qu'incombe la lourde tâche de "casser l'aiguille " réunissant les deux pans de l'ékerhei $^{12}$. Rude épreuve, selon tous nos informateurs, car il faut alors, devant toute l'assemblée réunie, se montrer à la hauteur du choix féminin, être assez fort pour briser sans hésitation l'aiguille, prendre soin de ne point blesser la jeune fille, etc. Une fois cette opération terminée, tous les hommes présents se doivent d'offrir un cadeau à la jeune fille qui a désormais le statut d'une femme.

L'élu qui a eu le privilège de "casser l'aiguille " devant se montrer alors le plus génércux de tous. Le cadeau qu'il offre, désigné par le terme ahayuf, est bien souvent un bel ékerhei ${ }^{13}$.

Ainsi, dans les rituels qui scandent deux moments clés de la vie d'une femme interviennent les stenfassen. Or, dans l'A haggar, ces étapes sont pour toute femme un début et une fin, une ouverture et une fermeture. Revêtir l'ékerhei, c'est comme nous l'avons vu - quitter le statut d'adolescente et devenir une femme. C'est aussi entrer par là même dans l'état d'asri que l'on peut traduire à la suite du père de Foucauld par l'expression "liberté de mœurs». Plus précisément le verbe esri (dont le premier sens est " courir à bride rendue ») signifie

... qu'on fait des actes de liberté de mœurs quelconques, depuis les moindres, tels que des attouchements un peu libres, jusqu'aux plus graves ... esri signific aussi qu'on n'est pas actuellement marié, qu'on ne vit pas dans la pénitence, et que par conséquent, on est toujours prêt à faire des actes de liberté de mœurs avec toute personne de sexe différent qui ne déplaît pas. Les Kel Ahaggar connaissent trois états de vie pour les hommes et les femmes qui par leur âge sont en état de courtiser ou d'être courtisé : être marié ; vivre dans la pénitence, vivre dans l'ćtat de liberté de mœurs. Aucun homme ni aucune femme ne vit dans la pénitence dans l'Ahaggar; tous les hommes et toutes les femmes qui ne sont pas actuellement mariés vivent dans l'état de liberté de mœurs (Foucauld 1952, IV : 1 854).

11. (suite) qu'a comtrario seule la prise du voile par les jeunes hommes y dommait lieu à un cérémonial particulier. On notera également que chez cux " les premieres manifestations de la féminité n'imposent pas que l'adolescente prenne le voile " (Casajus $1987: 320)$.

12. Nombre d’auteurs ont signalé que la prise du voile donnait lieu a une fête dans l'Ahaggar. Ils ont tous note le lien étroit entre apparition des premieres menstrues et prise de l'ékerhei (par exemple, Benhavera 1908:5, Foley 1930:211), mais aussi dans l'Ajjer (Campana-Norran 1959:506) ou l'Adrar des ffoghas (Cortier $1908: 309$. Chabrolles $1990: 123)$. Mais aucun, a notre connaissance, na decrit en détails ee rituel et l'épisode de l'aiguille notamment n'avait pas été relevé.

13. I.e terme ahayuf désigne en premier lieu "la chevelure non tressée ... longue ou courte d'un homme ou d'unc femme " (Foucauld 1951, II : 545). Mais ce terme désigne aussi le "cadeau de parrainage" offert par la personne dont le nom a été donné a un nouveau-né qui devient ainsi son homonyme (anemerra). Si ce second sens a plusieurs fois éte relevé (Barrere 1986), tel n'est point le cas du cadeau offert par l'homme qui casse l'aiguille à celle qui sera désormais une femme. 
Porter l'ékerhei, c'est devenir une femme courtisable, c'est avoir accès - et alors seulement - aux réunions galantes (ahâl), c'est entrer dans le jeu subtil des relations amoureuses si importantes chez le Kel-Ahaggar ${ }^{14}$.

Dès lors, les jeunes gens pourront s'empresser autour de cette " nouvelle venue " et rivaliser de galanterie pour obtenir ses faveurs. Un poème recueilli par le père de Foucauld (1925: 241) énonce clairement ce changement :

Celle qui depuis longtemps était regardée comme l'ornement futur des réunions galantes, qui a le cou de la pouliche, les yeux de l'antilope,

lorsqu'elle a mis pour la première fois l'asekref, elle a conquis tous les cœurs, dès que les jeunes gens ont pu aller la voir ${ }^{15}$.

Cet état d'asri cesse dès lors qu'une femme se marie. En ce sens, tout mariage ravit une femme aux jeunes hommes qui pouvaient prétendre obtenir ses faveurs.

C'est là un thème qui revient fréquemment dans les poésies touarègues (par exemple, Foucauld 1925, I : 183 et II : 258) ainsi que dans les chants (âléwen) qui accompagnent les différents moments du rituel du mariage. Chants qui bien souvent invitent les jeunes gens à la résignation :

S'il vous plaît, cessez d'en vouloir [au marié] jeunes gens,

S'il y a quelqu'un qui aimait [la mariée], à présent ça ne sert plus à rien,

S'il a un malaise, qu'il verse un peu d'eau sur son cœur (Mecheri $1986: 125$ ).

Ainsi, le mariage clôt l'époque ouverte par la prise du voile. Lors de celle-ci un homme « cassait l'aiguille » permettant ainsi à la jeune fille d'entrer dans le groupe des femmes. La connotation sexuelle de ce geste étant évidente y compris aux yeux des Kel-Ahaggar (hommes et femmes) qui ont bien voulu nous décrire cette cérémonie.

Quand cette même femme se marie, elle clôture cette époque de "liberté de mœurs " en offrant le même objet qui avait symbolisé son entrée en asri aux iklan qui, sur le chemin vers la tente nuptiale, lui barrent le passage. Le fait que ce soit à des « esclaves » que les stenfassen soient offertes n'a rien d'étonnant ${ }^{16}$. Vu la connotation sexuelle relevée ci-dessus, il serait inconvenant en effet qu'une femme lors même de son mariage offre des aiguilles à un homme de sa condition.

14. Ce rapport entre prise du voile et sexualité serait direct et immédiat si l'on n'en croit $\mathrm{M}$. Chabrolles en ce qui concerne l'Adrar des Ifoghas. Dans une langue imagée, cet auteur explique en effet que " pour la Damoiselle targuia, cet événement est un peu ce que le premier bal est pour une Européenne : une entrée officielle dans le monde... La jeune fille fixe son choix sur un sigisbée qui, souvent, lui tient compagnie jusqu'à l'aube. Ainsi, pour une jeune fille targuia, " prendre le voile " équivaut à jeter son bonnet par-dessus les moulins " (Chabrolles $1990: 123$ ). Pratique assez semblable, semble-til, dans l'Ajjer (Campana-Morvan 1959: 506).

15. A sekref désigne un ornement de chevelure porté en certaines circonstances par les femmes. Surtout, "du jour où une femme met l'asekref, c'est-à-dire déclare publiquement qu'elle est pubère, si elle n'est pas mariée, elle entre dans l'état d'asri et les hommes lui font des visites de galanterie " (Foucauld 1925: 241). Cf. aussi Drouin $1976: 295$.

16. De même, le fait que les aiguilles soient offertes à des hommes ne peut surprendre et ce pour deux raisons. D'abord, parce qu'à la différence du monde occidental, les aiguilles et la couture n'ont pas chez les Touaregs Kel-A haggar et d'une manière plus générale dans le monde berbère (Laoust 1930 : 185-188) une connotation spécifiquement féminine. Nombre de Kel-Ahaggar ont toujours sur eux cet instrument et il est fréquent de rencontrer l'un d'eux en train de coudre soit sa tunique (eraswey) soit son pantalon (ekerbei). De plus les relations interpersonnelles (et le système d'attitudes où interviennent les notions de réserve, honte, pudeur...) font qu'il serait inconvenant, voire humiliant, pour un 
En faisant ce don à des iklan, c'est-à-dire à des hommes qui, vu l'extrême hiérarchisation sociale de la société Kel Ahaggar, ne peuvent prétendre entretenir une relation avec elle ${ }^{17}$, elle abolit la portée réelle de son acte.

Il n'en reste pas moins que par ce geste fort symbolique qui consistait à «casser l'aiguille " retenant son ékerhei, un homme signifiait à la jeune femme que, quittant l'enfance, elle entrait dans une période de "liberté » (asri) et que par ce don des stenfassen, la même femme semble reconnaître que pour accéder à son nouveau statut d'épouse, elle clôt cette période.

En l'empêchant d'atteindre la tente nuptiale, les iklan attendent certes un cadeau de celle qui, à plus ou moins longue échéance, sera amenée à quitter le campement. Mais si le don de la nouvelle mariée prend la forme d'une aiguille dont la brisure avait signifié son entrée en asri, il indique alors la rupture avec son passé. Rupture nécessaire si elle veut accéder au nouvel état que son mariage vient de consacrer.

Saint-Denis

Université de La Réunion

\section{Bibliographie}

BARRÈRE, G. 1986. "Ahayuf ", Encyclopédie berbère III : 312-313.

BENHAZERA, M. 1908. Six mois chez les Touaregs du Hoggar. Alger, Jourdan.

CALAME-GRIAUlE, G. 1981. "Blanche-neige au Soleil », in : Itinéraires II, Mélanges offerts à $P$. $-F$. Lacroix. Paris, Société des africanistes : 61-78 (Mémoires de la Société des africanistes).

CAMPANA, J. et R. MORVAN 1959. "Les Touaregs Ajjer et Fort-Polignac, Étude géographique, historique et médicale ", Archives de l'Institut Pasteur d'Algérie XXXVII (3) : 474-547.

CASAJUS, D. 1987. La tente dans la solitude. Cambridge et Paris, Maison des sciences de l'homme et Cambridge University Press.

CHABRolles, M. 1990. Les hommes du voile. Paris, Cistre.

CHATELARD, A. 1990. "Azalay ", Encyclopédie berbère VIII : 1 205-1 206.

CORTIER, M. 1908. D'une rive à l'autre du Sahara. Paris, Larose.

DOUTTÉ, E. 1984. Magie et religion dans l'Afrique du Nord. Paris, J. Maisonneuve et P. Geuthner.

DROUIN, J. 1976. "Contraintes matrimoniales et compensations ludiques dans la société touarègue ", in : Production, pouvoir et parenté dans le monde méditerranéen de Sumer à nos jours. Paris, Geuthner : 293-304.

16. (suite) homme de donner ses vêtements à laver ou à coudre aux femmes. Il s'ensuit que ces tâches sont souvent assumées par les hommes eux-mêmes ou qu'ils s'en dèchargent, quand l'occasion se présente, sur une femme avec qui ils entretiennent des rapports détendus. Enfin, si comme nous le suggérons les stenfassen ont dans les deux rituels envisagés une forte connotation sexuelle, elles ne peuvent apparaître que dans une mise en scène où élément masculin et élément féminin sont mis en relation. 17. Il est extrêmement mal vu, dans l'Ahaggar, qu'une femme fréquente ou épouse un homme de statut social inférieur et, plus encore, qu'une femme libre (noble ou vassale) ait une liaison avec un akli. L'hypogamie féminine est donc extrêmement rare et toujours fortement réprouvée (Foucauld 1925, I : 317). 
IOIEY, H. 1930. "Mœurs et médecine des Touaregs de l'Ahaggar ", Archives de l'Institut Pasteur d'Algérie VIII (2) : 168-287.

louchuld, Ch. de 1925. Poésies touarègues. Dialecte de l'Ahaggar. Paris, Leroux, $2 \mathrm{t}$.

- 1951. Dictionnaire touareg-français. Paris, Imprimeric nationale, 4 vol.

IOUCAUI.D, Ch. de et A. de CAIASSANTI-MOTYIINSKI. 1984. Textes touaregs en prose, édition critique avec traduction par S. Chaker, H. Claudot et M. Gast. Aixen-Provence, Edisud.

(iAI.I.ANID-PERNET, P. 1978. "Images et image de la femme dans les poésies touarègues de l'Ahaggar », $B L O A B$ 9: 5-52.

(iAST, M. 1973. "Le don des sandales dans la cérémonie du mariage chez les KelAhaggar ", Actes du $I^{\text {er }}$ Congrès des cultures méditerranéennes d'influence arabo-berbère. Alger, SNED : 522-527.

L.AOUST, E. 1930. "L'habitation chez les transhumants du Maroc central ", Hesperis $\mathrm{X}: 151-253$.

- 1993. Noces berbères, les cérémonies du mariage au Maroc. Aix-en-Provence, Edisud/La Boîte à documents.

I.EACH, E. 1980. L'unité de l'homme et autres essais. Paris, Gallimard.

I.HOTE:, H. 1951. Dans les campements touaregs. Paris, Amiot-Dumont.

- 1984. Les Touaregs du Hoggar. Paris, A. Colin.

MLCHERI SAADA, N. 1986. La Musique de l'Ahaggar, thèse d'ethnologie. Paris, université Paris $\mathrm{X}$.

NICOIAISt:N, J. 1961. «Essai sur la religion et la magie touarègues », Folk 3 : 113-162.

- 1962. Structures sociales et politiques des Touaregs de l'Aï et de l'Ahaggar. Niamey, Ifan/CNRS (Études nigériennes 7).

PANDOI.II, P. 1989. Entre tente et maison : l'ekkeber. Saint-Denis, université de la Réunion, mémoire de maîtrise d'ethnologie.

- 1990. Première approche d'une société nomade du Sud algérien : les Touaregs Kel-Ahaggar. Saint-Denis, université de la Réunion, DEA d'anthropologie.

RECiNIE:R, J. 1961. "Mariage touareg ", Bulletin de liaison saharienne 42 : 163-171.

VI:R.MAI.K., P. 1926. Au Sahara pendant la guerre européenne (correspondance et notes publiées par A. Bernard). Alger.

WeSTERMARCK, E. 1921. Les cérémonies du mariage au Maroc. Paris, Leroux.

PAlil PAND)LH, doctorat en ethnologie, Centre d'anthropologie généralisće, Université de Saint-Denis, 9700() La Réunion. 